\title{
Protective Effect of Tat PTD-Hsp27 Fusion Protein on Tau Hyperphosphorylation Induced by Okadaic Acid in the Human Neuroblastoma Cell Line SH-SY5Y
}

\author{
Sunghyun Choi ${ }^{1} \cdot J^{1}$ Hoon $\mathrm{Oh}^{1} \cdot$ Hyeseon $\mathrm{Kim}^{1} \cdot$ So Hee Nam ${ }^{1} \cdot$ Jeehae Shin $^{1} \cdot$ \\ Jong-Sang Park ${ }^{1}$
}

Received: 3 February 2015/ Accepted: 21 April 2015/Published online: 20 May 2015

(C) The Author(s) 2015. This article is published with open access at Springerlink.com

\begin{abstract}
Alzheimer's disease (AD) is an age-related disorder that causes a loss of brain function. Hyperphosphorylation of tau and the subsequent formation of intracellular neurofibrillary tangles (NFTs) are implicated in the pathogenesis of AD. Hyperphosphorylated tau accumulates into insoluble paired helical filaments that aggregate into NFTs; therefore, regulation of tau phosphorylation represents an important treatment approach for AD. Heat shock protein 27 (Hsp27) plays a specific role in human neurodegenerative diseases; however, few studies have examined its therapeutic effect. In this study, we induced tau hyperphosphorylation using okadaic acid, which is a protein phosphatase inhibitor, and generated a fusion protein of Hsp27 and the protein transduction domain of the HIV Tat protein (Tat-Hsp27) to enhance the delivery of Hsp27. We treated Tat-Hsp27 to SH-SY5Y neuroblastoma cells for $2 \mathrm{~h}$; the transduction level was proportional to the Tathsp27 concentration. Additionally, Tat-Hsp27 reduced the level of hyperphosphorylated tau and protected cells from apoptotic cell death caused by abnormal tau aggregates. These results reveal that Hsp27 represents a valuable protein therapeutic for AD.
\end{abstract}

Sunghyun Choi and Jae Hoon Oh contributed equally to this work.

Electronic supplementary material The online version of this article (doi:10.1007/s10571-015-0199-1) contains supplementary material, which is available to authorized users.

Jong-Sang Park

pfjspark@snu.ac.kr

1 Department of Chemistry, Seoul National University, 599 Gwanak-ro, Gwanak-gu, Seoul 151-747, Republic of Korea
Keywords Tau protein - Hyperphosphoylation · Alzheimer's disease · Heat shock protein 27 . Protein transduction domain

\section{Introduction}

Alzheimer's disease (AD) is a progressive neurological disorder that causes memory loss. Various pathological hallmarks of AD include synaptic and neuronal loss, amyloid plaques primarily composed of the 42-residue hydrophobic $\beta$-amyloid peptide (A $\beta$ ) (Selkoe 2002), and neurofibrillary tangles (NFTs) composed of aggregates of hyperphosphorylated tau, which is a microtubule-associated protein (Kosik and Shimura 2005). Amyloid plaques and NFTs are considered as the primary factors involved in the pathogenesis of AD (Medeiros et al. 2010). Although previous studies have primarily focused on the role of $\beta$-amyloid peptides, recent studies on the role of tau in $\mathrm{AD}$ pathogenesis have indicated that hyperphosphorylated tau aggregates into insoluble paired helical filaments (PHFs), which induce neuronal dysfunction (Santa-Maria et al. 2012). Moreover, these two pathological hallmarks demonstrate synergistic effects on synaptic dysfunction (Crimins et al. 2013; Chabrier et al. 2014).

As a microtubule-associated protein, aggregates of normal tau protect cells against toxic hyperphosphorylation, although neuronal death occurred following a period of survival (Hernandez and Avila 2007). However, tau is not only abnormally phosphorylated but also aggregates into insoluble forms such as PHFs and NFTs (Andorfer et al. 2003; Citron 2010) in various AD mouse models (Götz et al. 2001; Denk and RichardWade-Martins 2009; Baglietto-Vargas et al. 2014). Therefore, an approach that reduces the level of hyperphosphorylated tau would 
represent a valuable treatment for AD (Buée et al. 2000). Tau can bind heat shock proteins (HSPs), which trigger the recruitment of CHIP (which is a co-chaperone that exhibits E3 activity), to the complex. CHIP has been shown to interact with tau, appears to work in concert with both $\mathrm{Hsp} 70$ and Hsp90 in degrading toxic tau species (Saidi et al. 2015). CHIP induces the ubiquitination of tau and activates its degradation when tau is defective (Salminen et al. 2011). In addition, The BAG2/Hsp70 complex is tethered to the microtubule and this complex can capture and deliver Tau to the proteasome for ubiquitin-independent degradation (Carrettiro et al. 2009). Curcumin also downregulated the levels of phosphorylated tau, which may be potentially attributed to the curcumin-induced upregulation in BAG2 levels in the neurons (Patil et al. 2013). Hsp27 directly associates with hyperphosphorylated tau or PHFs and regulates cell survival by eliminating tau aggregates (Shimura et al. 2004b).

Heat shock proteins are induced in response to cellular stress as molecular chaperones that inhibit protein aggregation (Read and Gorman 2009). HSPs can prevent apoptosis and increase cell viability during cellular stress (Shimura et al. 2004a; Kwon et al. 2007). HSPs are also critical regulators of normal neural physiological function and cell stress responses (Stetler et al. 2010; Arrigo 2013; Song et al. 2014). However, the therapeutic effects of exogenous Hsp27 on disease models have not been investigated. Therefore, in this study, we investigated whether Hsp27 could reduce hyperphosphorylated tau in AD-induced SH-SY5Y cells.

To more effectively deliver Hsp27 into cells, we combined the HIV protein transduction domain (PTD) Tat with the Hsp27 protein (Tat-Hsp27). HIV Tat (11 residues, YGRKKRRQRRR) can rapidly transduce into cells (Becker-Hapak et al. 2001) and deliver full-length proteins into cells (Green et al. 2003). Tat-Hsp27 effectively reduced the phosphorylation of tau and rescued the cell death caused by abnormal tau aggregates. Therefore, our study suggests that Tat-Hsp27 may represent a potential protein therapeutic for tau-induced neurodegeneration.

\section{Materials and Methods}

\section{Construction of the Expression Vector}

We designed a bacterial expression vector (His6-TatHsp27) containing hexahistidine leader sequence, 11-amino acid Tat PTD sequence (YGRKKRRQRRR), and Hsp27 protein sequence. The Hsp27 fragment (BD Bioscience) was generated by PCR using human cDNA as a template and oligonucleotide primers containing BamHI and XhoI restriction sites. The pET-28a vector (Novagen) and the Tat PTD were digested with NdeI and BamHI and ligated. The ligated vector was transformed into chemically competent DH5 $\alpha$ E. coli cells (Enzynomics, Korea). After purification of the plasmid containing the Tat PTD, the plasmid and the Hsp27 fragment were digested with BamHI and XhoI and ligated. We also designed pET28a vector containing Hsp27 (wt-Hsp27) genetically fused with a hexahistidine (His6) tag. The plasmid was constructed by digesting the pET28a vector with BamHI and XhoI restriction endonucleases and ligating the Hsp27 fragment digested with the same restriction enzymes into the cut vector. The recombinant plasmids (pET28a-Tat-Hsp27, pET28a-Hsp27) were transformed into the Escherichia coli strain BL21 (DE3) for protein expression (Enzynomics, Korea).

\section{Expression and Purification of Hsp27}

The recombinant plasmid (His6-Tat-Hsp27) was transformed into the E. coli strain BL21(DE3) for protein expression. Transformed cells were plated on an LB agar (Merck) plate containing kanamycin (Sigma-Aldrich) and incubated overnight at $37^{\circ} \mathrm{C}$. Two-hundred milliliters of LB medium containing $1 \mathrm{mM}$ kanamycin was inoculated with a single colony and incubated overnight at $37^{\circ} \mathrm{C}$ with shaking at $200 \mathrm{rpm}$. The following day, $1 \mathrm{~L}$ of LB medium was inoculated with this preculture and incubated at $37{ }^{\circ} \mathrm{C}$ until an $\mathrm{OD}_{600}$ of 0.5 was reached. Protein expression was induced by the addition of Isopropyl $\beta$-D-1thiogalactopyranoside (MB cell, Korea) at a final concentration of $1 \mathrm{mM}$, and the cells were incubated at $37^{\circ} \mathrm{C}$ for an additional $4 \mathrm{~h}$. The cells were centrifuged at $6000 \mathrm{rpm}$ for $15 \mathrm{~min}$ at $4{ }^{\circ} \mathrm{C}$ and resuspended in binding buffer $(20 \mathrm{mM}$ Tris- $\mathrm{HCl}, 500 \mathrm{mM} \mathrm{NaCl}, 35 \mathrm{mM}$ imidazole, $\mathrm{pH}$ 7.5). Cells were lysed by sonication on ice using a sonicator (Sonics Vibra-Cell VCX 750, Sonic \& Materials Inc., USA) with 1 -s pulses and 8 -s pauses for $30 \mathrm{~min}$. After sonication, the lysates were centrifuged at $10,000 \mathrm{rpm}$ for $15 \mathrm{~min}$. The clarified lysate was loaded onto a pre-equilibrated HisTrap HP column (GE Healthcare). His-tagged Tat-Hsp27 protein was eluted with elution buffer $(20 \mathrm{mM}$ Tris- $\mathrm{HCl}, 500 \mathrm{mM} \mathrm{NaCl}, 1 \mathrm{M}$ imidazole, $\mathrm{pH}$ 7.5). His-tagged Tat-Hsp27 was further purified using size exclusion chromatography on a HiLoad 16/600 Superdex 200 prep grade column (GE Healthcare) using $20 \mathrm{mM}$ Tris- $\mathrm{HCl}(\mathrm{pH} 7.5)$ and $100 \mathrm{mM}$ $\mathrm{NaCl}$. Wt-Hsp27 purification was performed in the same manner as in the purification step above. For the detection of Tat-Hsp27 delivery into cells, we conjugated FITC to Tat-Hsp27 using an FITC labeling kit (Thermo Scientific). The concentration of protein was determined using BCA protein assay kit (Pierce). 


\section{Labeling of wt-Hsp27, TAT-Hsp27}

To eliminate any primary amines or ammonium ions from previous buffer, the buffers for both wt-Hsp27 and TATHsp27 were exchanged with $50 \mathrm{mM}$ sodium borate of $\mathrm{pH}$ 8.5 using Amicon ultra centrifugal filters. Two-hundred thirty micrograms of the prepared protein was added to a vial of FITC Reagent $(50 \mu \mathrm{g})$. The reaction mixture was incubated for $60 \mathrm{~min}$ at room temperature protected from light. After adding the mixtures to the spin columns, they were centrifuged to remove any excess FITC at $1000 \times g$ for $1 \mathrm{~min}$.

\section{Cell Culture}

SH-SY5Y human neuroblastoma cells were grown in Roswell Park Memorial Institute 1640 medium supplemented with $10 \%$ fetal bovine serum and $1 \%$ antibioticantimycotic under an atmosphere of $5 \% \mathrm{CO}_{2}$ and $95 \%$ air (all from WELGENE). The medium was refreshed every three days. Cells below passage 24 were used for experiments. We chose the SH-SY5Y cell line because these human neuroblastoma cells express constantly endogenous tau.

\section{Western Blot Analysis}

For Western blot analysis, the cells treated with Tat-Hsp27 and okadaic acid were washed in DPBS and harvested in RIPA buffer (Pierce). To prevent from further phosphorylation, we added Protease inhibitor cocktail and phosphatase inhibitor cocktail composed of sodium orthovanadate, sodium molybdate, sodium tartrate, and imidazole (both from Sigma-Aldrich) into the cell lysates. Lysates were then centrifuged at $15,000 \times g$ for $15 \mathrm{~min}$ at $4{ }^{\circ} \mathrm{C}$. The supernatant was separated in a $12 \%$ SDS-PAGE gel, and the proteins were transferred to a nitrocellulose membrane. After blocking in $5 \%$ skim milk/TBST (Trisbuffered saline and $0.1 \%$ Tween $20, \mathrm{pH} 7.5$ ), the membrane was washed in TBST three times (15 min each) and incubated with primary antibody overnight at $4{ }^{\circ} \mathrm{C}$. The membrane was subsequently washed and incubated with the appropriate secondary antibody (HRP-conjugated goat antimouse and goat anti-rabbit) for $2 \mathrm{~h}$ at room temperature. Protein signals were developed with ECL Western Blotting Detection Reagent (Amersham Pharmacia Biotech, Piscataway, NJ, USA) exposed to X-ray film. Band intensities were calculated with the Image $J$ software, and the protein band of interest was normalized to $\beta$-actin. Then, tau hyperphosphorylation was normalized to the total tau as a ratio, pTau/Total tau. Peroxidase-linked anti-rabbit and anti-mouse IgGs were purchased from Santa Cruz Biotechnology (USA). Rabbit polyclonal anti-tau phosphoserine 199/202 antibody and mouse monoclonal anti-tau-1 antibody were obtained from Millipore (USA). Anti-human total tau monoclonal antibody (HT 7), rabbit anti-Hsp27 polyclonal antibody, and anti Phospho-PHF-tau (detected phospho-S202, -T205) monoclonal antibody (AT 8 ) were purchased from Pierce Biotechnology (USA). AntiHIV1 tat monoclonal antibody was purchased from Abcam (USA). Synthesized tat peptides (YGRKKRRQRRR) were purchased from PEPTRON (Korea).

\section{Immunocytochemistry}

The SH-SY5Y cells were seeded on 2-well slides (Lab-Tek chamber, Nalge Nunc, NY) at a density of $5.0 \times 10^{5}$ per well, treated with $2 \mu \mathrm{M}$ Tat-Hsp27 for $2 \mathrm{~h}$, and then treated with $10 \mathrm{nM}$ okadaic acid for $14 \mathrm{~h}$. The cells were rinsed two times with PBS and fixed with $4 \%$ paraformaldehyde for $30 \mathrm{~min}$. The cells were subsequently permeabilized for 10 min with $0.5 \%$ Triton X-100 followed by three 5-min washes in PBS. To reduce nonspecific binding, we used PBS containing $0.5 \%$ bovine serum albumin as blocking buffer. After incubation with the blocking buffer for $1 \mathrm{~h}$, the cells were incubated with the primary antibody AT8 (anti Phospho-PHF-tau detected phospho-S202, -T205) for $4 \mathrm{~h}$, followed by three 5-min washes. The cells were subsequently incubated with goat anti-rabbit $(\mathrm{H}+\mathrm{L})$ FITCconjugated antibody. After washing with PBS, cells were imaged using an image restoration microscope (Applied Precision, USA).

\section{Cell Viability/Cytotoxicity Assay}

\section{A Cytotoxicity Assay was Performed}

Using a Cell Counting Kit-8 (Dojindo, Korea). SH-SY5Y cells were plated in a 96-well plate at $5.0 \times 10^{4}$ in $100 \mu \mathrm{L}$ of RPMI 1640 medium containing $10 \%$ FBS. After incubation for $48 \mathrm{~h}$, cells were treated with $0.5 \mu \mathrm{M}$ or $2 \mu \mathrm{M}$ Tat-Hsp27 for $2 \mathrm{~h}$. Subsequently, 10nM and 50nM okadaic acid were added for $6 \mathrm{~h}$ to induce abnormally phosphorylated tau. Ten microliters of CCK- 8 solution was added, and the cells were incubated at $37{ }^{\circ} \mathrm{C}$ for $2 \mathrm{~h}$. The absorbance at $450 \mathrm{~nm}$ was measured using a microplate reader (Molecular Devices Co., Menlo Park, CA).

\section{In Situ Terminal Deoxynucleotidyl Transferase dUTP Nick-End Labeling (TUNEL) Assay}

SH-SY5Y cells were seeded on a 2-well slide at a density of $5.0 \times 10^{5}$ per well, treated with $2 \mu \mathrm{M}$ Tat-Hsp27 for $2 \mathrm{~h}$, and then treated with $10 \mathrm{nM}$ okadaic acid for $14 \mathrm{~h}$. The cells were fixed by immersion in PBS containing $4 \%$ formaldehyde ( $\mathrm{pH}$ 7.4). After washing with PBS, the cells 
were permeabilized in $0.2 \%$ Triton $\mathrm{X}-100$ and washed again. After treatment with equilibration buffer, the cells were added to rTdT incubation buffer containing nucleotide mix. To terminate the reaction, saline sodium citrate buffer was added to the cells; nuclei were stained with DAPI and the mounting media used was Vectashield. The cells were analyzed under a fluorescence microscope at $520 \mathrm{~nm}$ (green fluorescence) and $460 \mathrm{~nm}$ (blue, DAPI).

\section{Statistical Analysis}

Statistical analysis was performed with the Statistical Package for the Social Sciences (SPSS, IBM Corporation, NY, USA). Statistical significance was determined by analysis of variance (ANOVA) with Tukey's HSD test and student's $t$ test. All results are presented as the mean \pm standard deviation, and statistical significance was considered at the $5 \%$ level.

\section{Results}

The sequence encoding Tat-Hsp27 fusion protein was cloned into the pET28a vector, which produces a recombinant protein with a hexahistidine tag (Fig. 1a) and purified using affinity purification and size exclusion chromatography. Fractions containing His-tagged TatHsp27 were identified using $12 \%$ SDS-PAGE analysis with Coomassie Brilliant Blue staining. His-tagged TatHsp27 in fractions 1-6 exhibited high affinity for the affinity matrix. The eluted His-tagged Tat-Hsp27 was further purified using size exclusion chromatography. Fractions containing His-tagged Tat-Hsp27 were identified using $12 \%$ SDS-PAGE with Coomassie Brilliant Blue staining. His-tagged Tat-Hsp27 eluted as a single peak from size exclusion chromatography, as confirmed by Western blot analysis (Fig. 1d), and migrated to a position slightly above the $25-\mathrm{kDa}$ molecular weight marker in $12 \%$ SDS-PAGE analysis. The purified recombinant Histagged wt-Hsp27 and Tat-Hsp27 was concentrated to 5.85 and $1.438 \mathrm{mg} / \mathrm{mL}$ as determined using the BCA assay, respectively.

To investigate whether Tat-Hsp27 could be effectively delivered into cells, we conjugated FITC to Tat-Hsp27. Image restoration microscopy indicated that FITC-TatHsp27 was efficiently delivered into SH-SY5Y cells following treatment for $2 \mathrm{~h}$ at a concentration of $2 \mu \mathrm{M}$, in contrast to $2 \mu \mathrm{M}$ wt-Hsp27. We also performed Western blot analysis to confirm the intracellular delivery of TatHsp27 and to determine whether its transduction is dependent on the Tat-Hsp27 concentration. SH-SY5Y cells were treated with Tat-Hsp27 for $2 \mathrm{~h}$ and were lysed using RIPA buffer. As shown in Fig. 2b, Hsp27 was present in the lysate of SH-SY5Y cells treated with 2 and $5 \mu \mathrm{M}$ TatHsp27, whereas equivalent concentrations of wt-Hsp27 did not transduce cells. Additionally, increasing the concentration of Tat-Hsp27 used to treat cells resulted in an increased level of delivered protein. Therefore, the recombinant Tat-Hsp27 can be delivered into cells in a concentration-dependent manner.

Because Hsp27 is known to have an effect on phosphorylation, we investigated whether Hsp27 can reduce the level of hyperphosphorylated tau, which is implicated in the pathogenesis of AD. SH-SY5Y cells were treated with 2 or $5 \mu \mathrm{M}$ Tat-Hsp27 for $2 \mathrm{~h}$, and the phosphorylation of normal tau was induced by the addition of 50 or $100 \mathrm{nM}$ okadaic acid. To quantify the relative phosphorylation, we normalized the level of hyperphosphorylated tau (p-tau) in Western blot analysis to the level of total tau using SigmaPlot software. Following treatment with 50 or $100 \mathrm{nM}$ okadaic acid for $2 \mathrm{~h}$, the level of hyperphosphorylated tau was approximately 2.4-fold or three-fold greater than the control group, respectively. Following treatment with $50 \mathrm{nM}$ okadaic acid, the level of $\mathrm{p}$-tau in cells pretreated with 2 and $5 \mu \mathrm{M}$ Tat-Hsp27 decreased with an increasing concentration of Tat-Hsp27 (Fig. 3), whereas equivalent concentration of tat peptide did not prevent tau hyperphosphorylation and oligomers (Supplementary Fig. S1). Tat-Hsp27 did not revert tau phosphorylation and aggregation into oligomers when added at the same time or after okadaic acid (as data not shown). Following treatment with $100 \mathrm{nM}$ okadaic acid, cells pretreated with $5 \mu \mathrm{M}$ TatHsp27 showed slightly higher levels of p-tau than cells pretreated with $2 \mu \mathrm{M}$ Tat-Hsp27. This finding suggest that when tau is hyperphosphorylated, the protective effect was better 2 than $5 \mu \mathrm{M}$ Tat-Hsp27 on the reduction in hyperphosphorylated tau. Therefore, we used $2 \mu \mathrm{M}$ Tat-Hsp27 in subsequent experiments. We also performed immunocytochemistry using an anti-p-tau antibody conjugated to FITC (Fig. 4). SH-SY5Y cells were treated with $2 \mu \mathrm{M}$ TatHsp27 for $2 \mathrm{~h}$ and were subsequently treated with $10 \mathrm{nM}$ okadaic acid for $14 \mathrm{~h}$. The level of p-tau was significantly decreased compared with treatment with okadaic acid alone $(n=3)$.

A cytotoxicity assay was performed to determine whether Tat-Hsp27 directly affected the cell death induced by hyperphosphorylated tau. We first evaluated the relative cell viability (RCV) in the presence of Tat-Hsp27 alone. As shown in Fig. 5a, treatment with Tat-Hsp27 alone did not alter the RCV of normal cells. In contrast, SH-SY5Y cells treated with 10 and $50 \mathrm{nM}$ okadaic acid for $6 \mathrm{~h}$ exhibited a decrease in $70.45,49.234 \%$ cell viability $(* p<0.05)$. When cells were treated with 0.5 or $2 \mu \mathrm{M}$ Tat-Hsp27 followed by treatment with 10 and $50 \mathrm{nM}$ okadaic acid, the cell viability increased to $92.44,90.78,76.89$, and $81.57 \%$, respectively $(* * p<0.01$; Fig. 5 b). Cells treated 
A

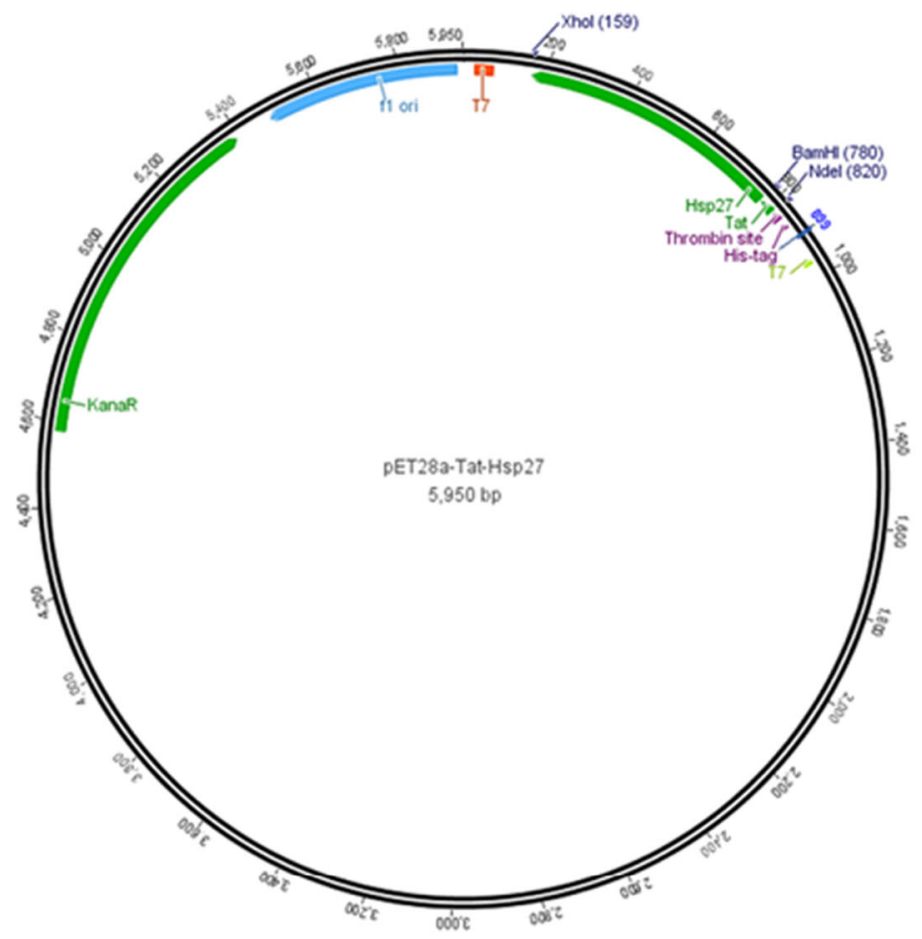

D

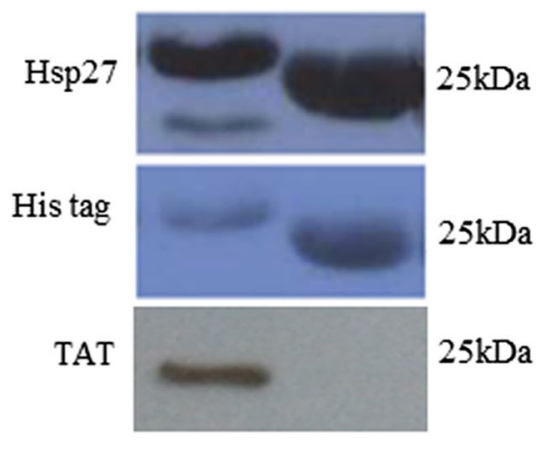

B
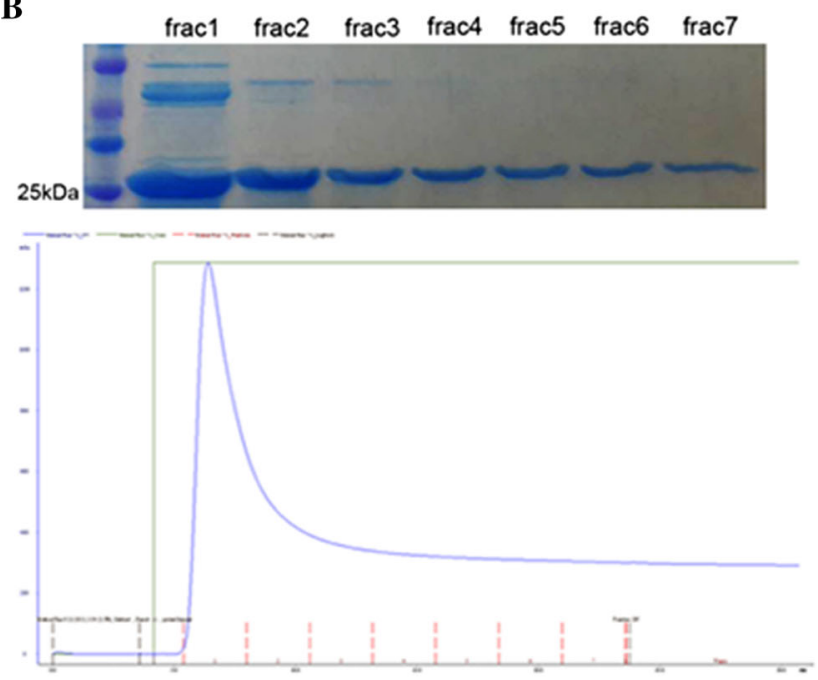

Buffer A: $20 \mathrm{mM}$ Tris(pH7.5), $500 \mathrm{mMNaCl} 35 \mathrm{mM}$ Imidazole Buffer B: $20 \mathrm{mM}$ Tris(pH7.5), $500 \mathrm{mM} \mathrm{NaCl}, 1 \mathrm{M}$ Imidazole Flow Rate: $5 \mathrm{~mL} / \mathrm{min}$, Buffer B Gradient: $100 \%$

Fig. 1 Purification of His-tagged Tat-Hsp27. a pET28a vector containing hexahistidine (His6) and Tat protein transduction domain and Hsp27 cDNA. b His-tagged Tat-Hsp27 was eluted from the affinity chromatography column using isocratic elution buffer

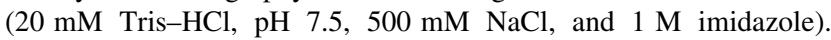
c His-tagged Tat-Hsp27 was further purified using size excluion

with 0.5 and $2 \mu \mathrm{M}$ Tat-HSP27 clearly showed a protective effect against cell death induced by hyperphosphorylated tau. Treatment with $10 \mathrm{nM}$ okadaic acid alone for $14 \mathrm{~h}$ greatly increased the number of TUNEL-positive cells,
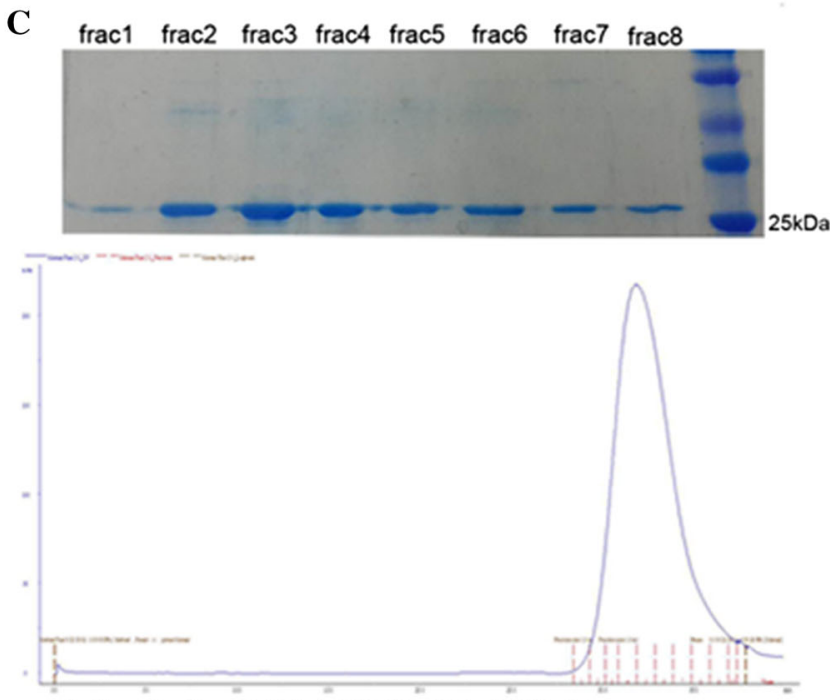

Buffer C: 20mM Tris(pH7.5), $100 \mathrm{mM} \mathrm{NaCl}$ Flow Rate: $1 \mathrm{~mL} / \mathrm{min}$

chromatography (20 mM Tris- $\mathrm{HCl}, \mathrm{pH} 7.5$, and $100 \mathrm{mM} \mathrm{NaCl}$ ) and eluted as a single peak. Fractions were analyzed using SDS-PAGE after each purification step. d Western blot analysis of purified Histagged Tat-Hsp27 using anti-His tag, anti-Hsp27, and anti-Tat antibodies

whereas the cells pretreated with $2 \mu \mathrm{M}$ Tat-Hsp27 exhibited decreased TUNEL positivity (Fig. 6). In the control group, apoptotic cells comprised $2.29 \%$ of the total number of cells. Following exposure to $10 \mathrm{nM}$ okadaic 
Fig. 2 Transduction of wt-Hsp27 and Tat-Hsp27 into SH-SY5Y cells.

a Immunocytochemistry. FITCconjugated wt-Hsp27 or TatHsp27 was delivered into cells and analyzed $2 \mathrm{~h}$ later. Image restoration microscopy indicates that Tat-Hsp27 was rapidly and efficiently delivered, in contrast to wt-Hsp27. Scale bar $30 \mu \mathrm{m}$. b Protein from cell lysates of SH-SY5Y cells treated with wt-Hsp27 or Tat-Hsp27 was analyzed using Western blot analysis. The protein expression was normalized to actin. c Experimental design for Tat-Hsp27 treatment in SH-SY5Y cells
A

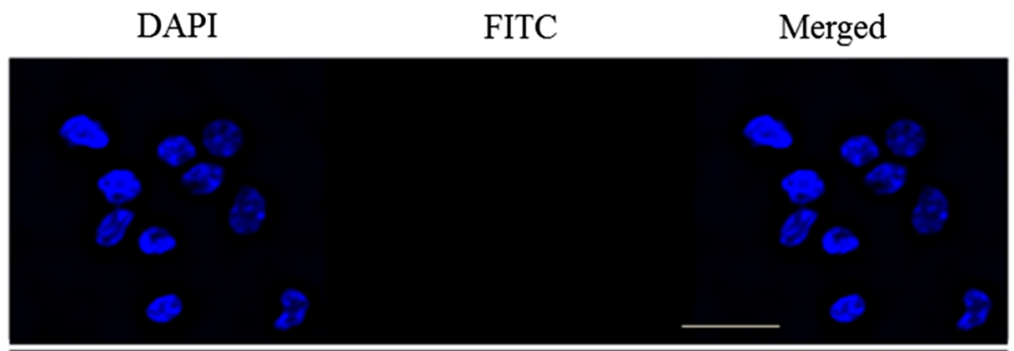

TAT-Hsp27

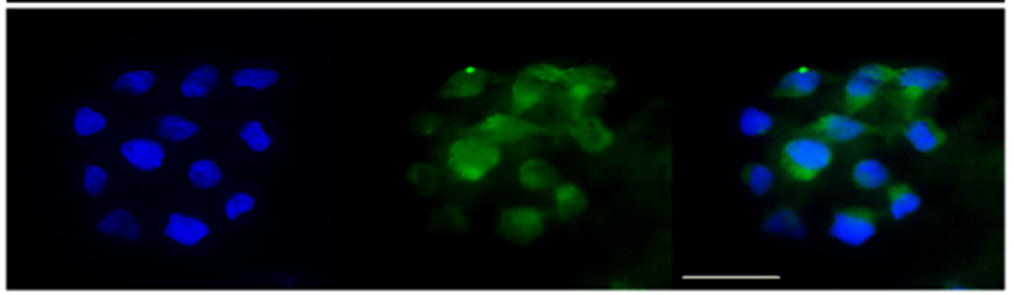

B

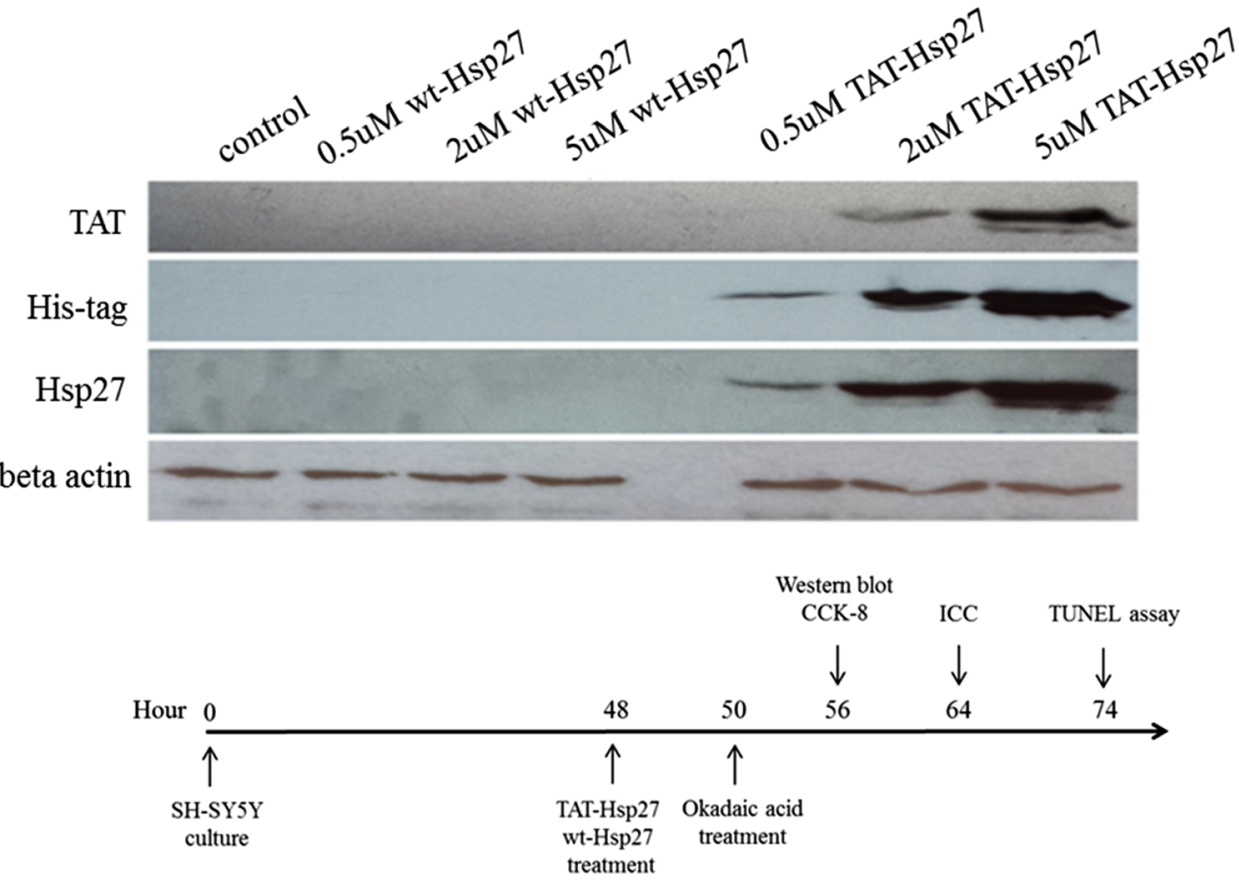

acid, the percentage of apoptotic cells increased to $17.84 \%$. However, this increase was inhibited by Tat-Hsp27 (4.56\%). Therefore, intracellular delivery of Tat-Hsp27 prevented the apoptotic cell death induced by hyperphosphorylated tau.

\section{Discussion}

In this study, the recombinant Tat-Hsp27 reduced the level of hyperphosphorylated tau induced by okadaic acid in SHSY5Y neuroblastoma cells and prevented the apoptosis induced by abnormal tau aggregates in our cellular model of AD. The effect of Hsp27 on phosphorylated tau has recently received increasing attention. However, few studies have examined the therapeutic effect of Hsp27 on hyperphosphorylated tau, which has been implicated in the pathogenesis of AD. We demonstrate that Hsp27 exhibits a protective effect on apoptotic cell death caused by pathological tau. This finding suggests that Hsp27 may represent a potential protein therapeutic for AD.

Tau stabilizes microtubules; however, tau mutations that result in its hyperphosphorylation lead to the formation of tau filaments that can form twisted ribbons or rope-like filaments (Kosik and Shimura 2005). Using phosphorylation-dependent monoclonal antibodies against tau, mass spectrometry, and sequencing, at least thirty phosphorylation sites have been reported (Bussiére et al. 2000). Because the phosphorylation of tau is regulated by various kinases, including proline-directed protein kinases and 
Fig. 3 Effect of Tat-Hsp27 on tau hyperphosphorylation. Western blot analysis of total tau (HT 7) and p-tau(AT 8), with $\beta$-actin as a loading control. $* p<0.05$ compared with the control. $* * p<0.05$ compared with okadaic acid alone. Values indicate mean \pm SD (Anova, Tukey's HSD test, $n=3$ per group)
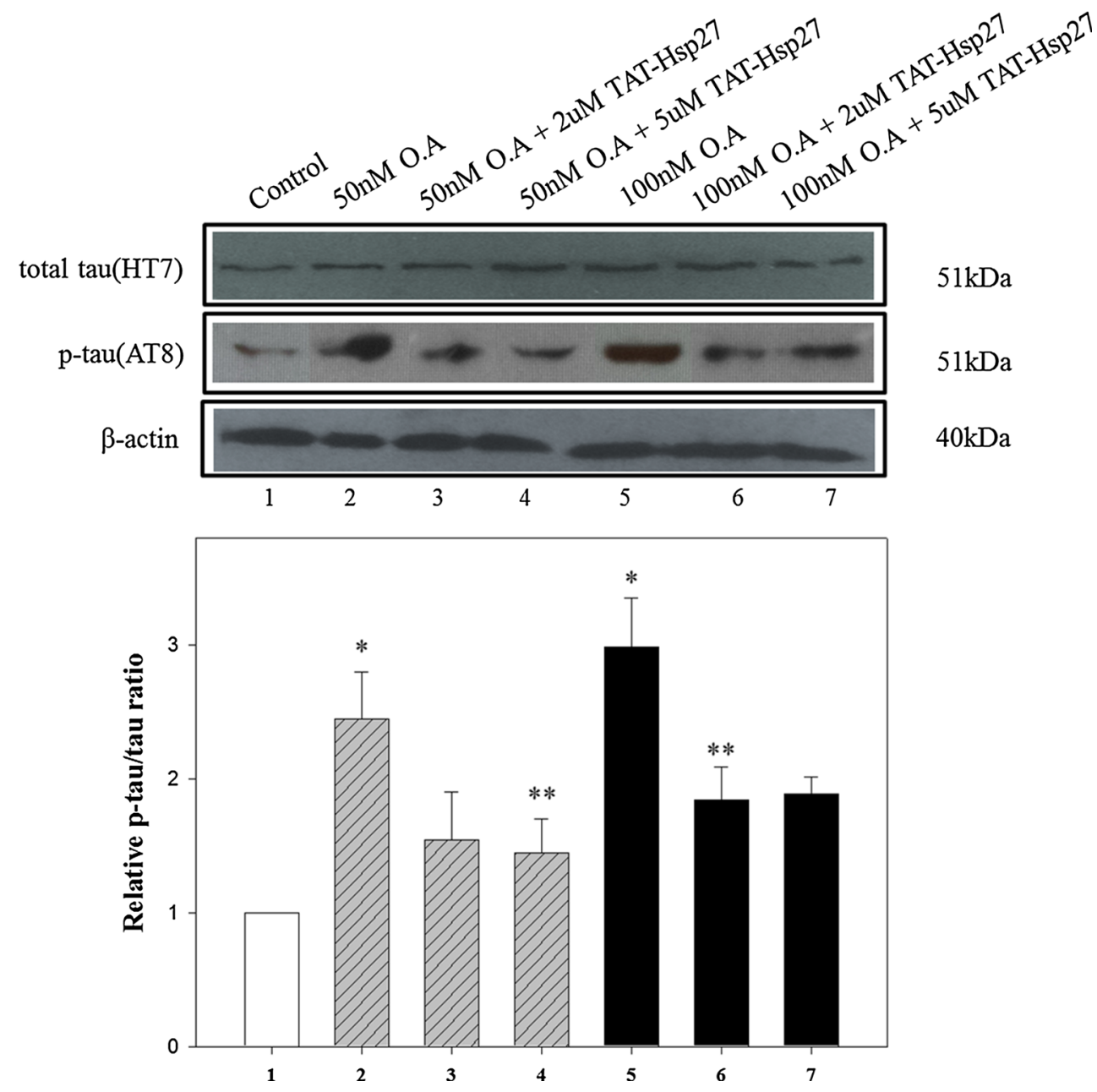

glycogen synthase kinase 3, and phosphatases, including Ser/Thr protein phosphatases 1, 2A, 2B (calcineurin) and $2 \mathrm{C}$, we used okadaic acid, which is a protein phosphatase inhibitor, to induce PHF-like hyperphosphorylation of tau (Zhang and Simpkins 2010). Hsp27 facilitates the degradation and prevents the aggregation of aberrant substrates independent of ATP or ubiquitination (Jakob et al. 1993; Shimura et al. 2004a, b). The mechanism by which Hsp27 prevents apoptosis could be from not only the interaction between Hsp27 and the pathologically hyperphosphorylated tau but also from Hsp27-mediated inhibition of procaspase-9 and caspase-3 (Concannon et al. 2003). PP2A dephosphorylates Hsp27 more effectively than that of PP1, which is weakly active. Many of the sites that are dephosphorylated by PP2A are phosphorylated by either GSK-3 $\beta$ or Cdk5. These include S199, S202, T205, S396, and S404 93-96. We used the AT8 antibody to detect phosphorylated tau at Ser202 and Thr205 (Goedert et al. 1995). Okadaic acid inhibits Ser/Thr protein phosphatases and can induce tau hyperphosphorylation and neurodegeneration (Kamat et al. 2013).
A recent study reported that hyperphosphorylation of tau leads to a 20-fold inhibition of the tau-tubulin binding affinity. This finding supports the critical role for tau in the pathogenesis of NFT-induced degeneration because the balance between kinases and phosphatases is disturbed in $\mathrm{AD}$, resulting in the disassociation of tau from microtubules and its subsequent aggregation (Wischik et al. 2014). In our previous study, we showed that hyperphosphorylated tau, but not the overexpression of normal tau alone, reduces the cell viability of the neuroblastoma cell line SH-SY5Y (Ahn et al. 2013). Thus, in the present study, a cellular model of $\mathrm{AD}$ was induced by the hyperphosphorylation of endogenous tau.

Moreover, we utilized the PTD of the HIV Tat protein to enhance the delivery of Hsp27, thereby enhancing its protective effect (Wadia and Dowdy 2005; Stetler et al. 2010). Conjugation with specific peptide sequences, which are termed PTDs or cell-penetrating peptides, improves the delivery of a range of agents, including antisense oligonucleotides, plasmids, microbeads, and liposomes, which suggests that these peptide sequences may represent 


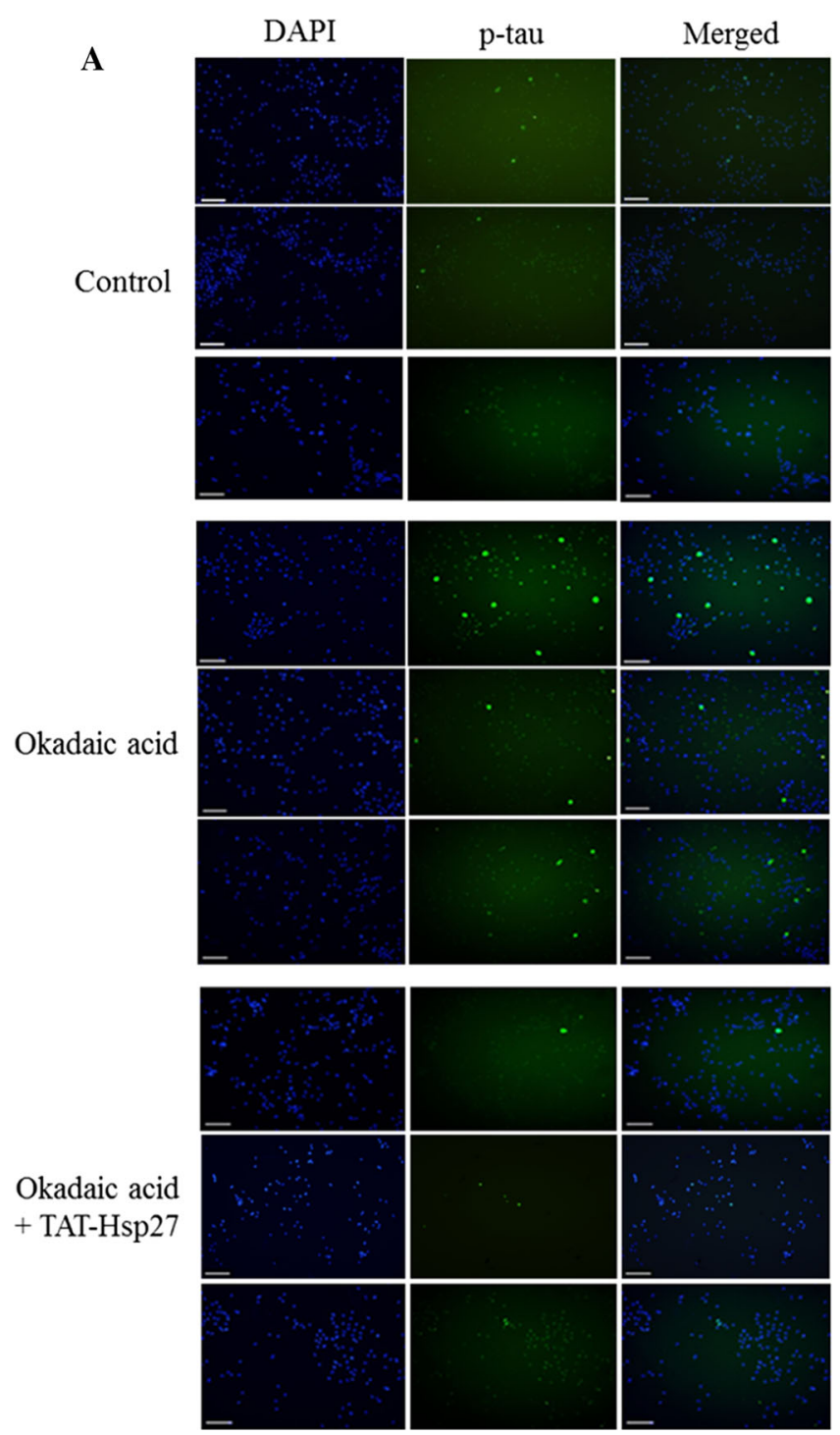

Fig. 4 Representative immunofluorescence images. a SH-SY5Y cells were treated with $2 \mu \mathrm{M}$ Tat-Hsp27 ( $2 \mathrm{~h}$ ) followed by $10 \mathrm{nM}$ okadaic acid $(14 \mathrm{~h})$. All cells were fixed and stained with primary antibodies against p-tau(AT 8) and the corresponding fluorescent secondary antibody. Green (FITC) indicates phosphorylated tau. Scale bar

a universal in vitro and in vivo cellular delivery system (Green et al. 2003). We confirmed that the fusion protein of Hsp27 and Tat PTD was delivered into normal human neuroblastoma SH-SY5Y cells and into SH-SY5Y cells containing hyperphosphorylated tau induced by okadaic acid treatment. To determine whether Tat-Hsp27 has a protective effect against okadaic acid-induced cell death, SH-SY5Y cells containing hyperphosphorylated tau were treated with the fusion protein. The levels of phosphorylated tau significantly decreased compared with the cells that were not treated with Tat-Hsp27, and the relative cell viability was enhanced. This result suggests that Tat-Hsp27 represents a potential protein therapeutic to reduce hyperphosphorylated tau.

\section{B}

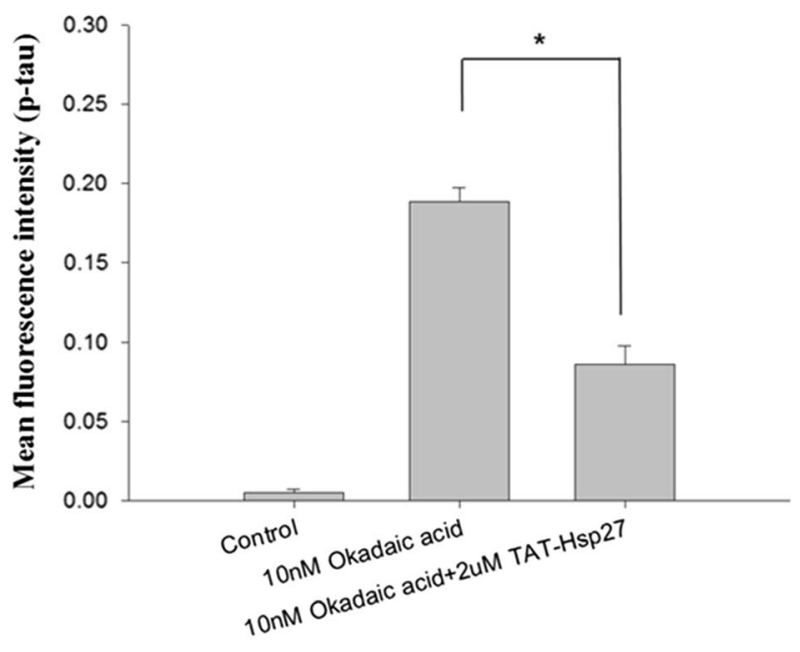

$100 \mu \mathrm{m}$. b Quantitation of the immunofluorescence intensity demonstrated that phosphorylated tau by Okadaic acid was decreased by treatment with Tat-Hsp27. Values indicate mean \pm SD (Anova, Tukey's HSD test, $n=3$ per group). $* p<0.05$ compared with okadaic acid alone

Protein delivery systems offer several advantages, such as the ease of production from synthetic or natural compounds which provoke low inflammatory response (Rogers and Rush 2012). Compared to other delivery systems, such as gene delivery utilizing plasmids or viral vectors, our recombinant Tat-Hsp27 has several advantages. The straightforward purification of Tat-Hsp27 is amenable to large-scale production. Moreover, Tat-Hsp27 is nontoxic and stable and can be readily delivered into cells.

As previously mentioned, PTDs can readily and rapidly deliver proteins into cells. Drugs to treat neurodegenerative diseases must be capable of penetrating the blood-brain barrier (BBB) to be effectively delivered (Ozsoy et al. 2009). However, greater than $98 \%$ of all potential CNS 


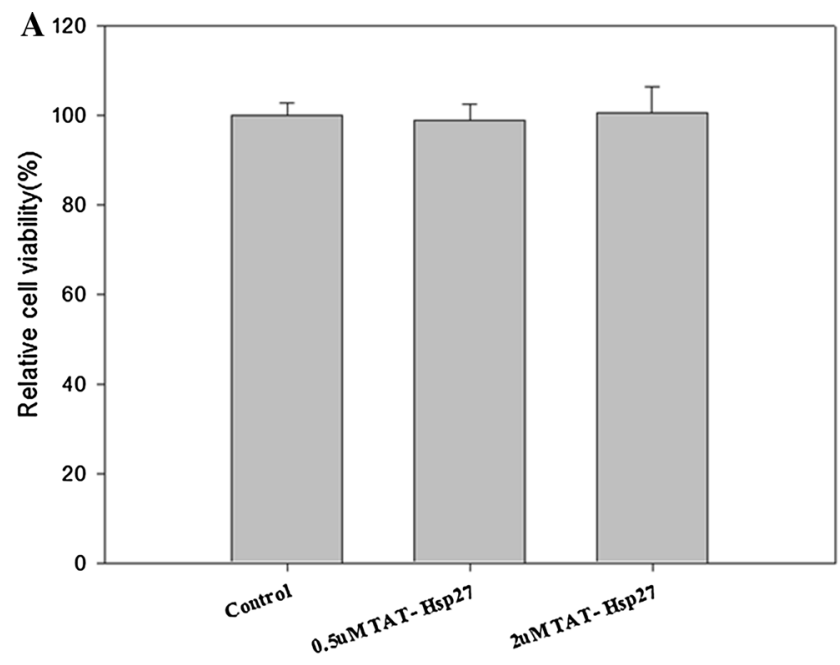

Fig. 5 Cytotoxicity assay using the Cell Counting Kit-8. SH-SY5Y cells were transduced with 0.5 or $2 \mu \mathrm{M}$ Tat-Hsp27 alone (a) or prior to treatment with 10 and $50 \mathrm{nM}$ okadaic acid $(\mathbf{b})$. The transduction of Tat-Hsp27 alone does not affect the cell viability. ${ }^{*} p<0.05$

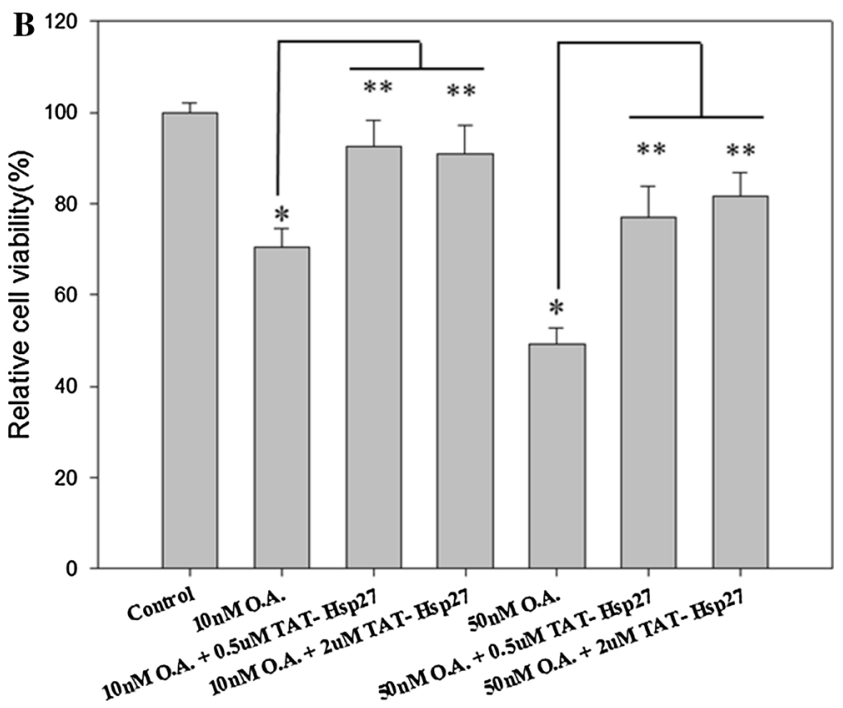

compared with the control. $* * p<0.01$ compared with okadaic acid alone. Values indicate mean $\pm \mathrm{SD}$ (Anova, Tukey's HSD test, $n=7$ per group)

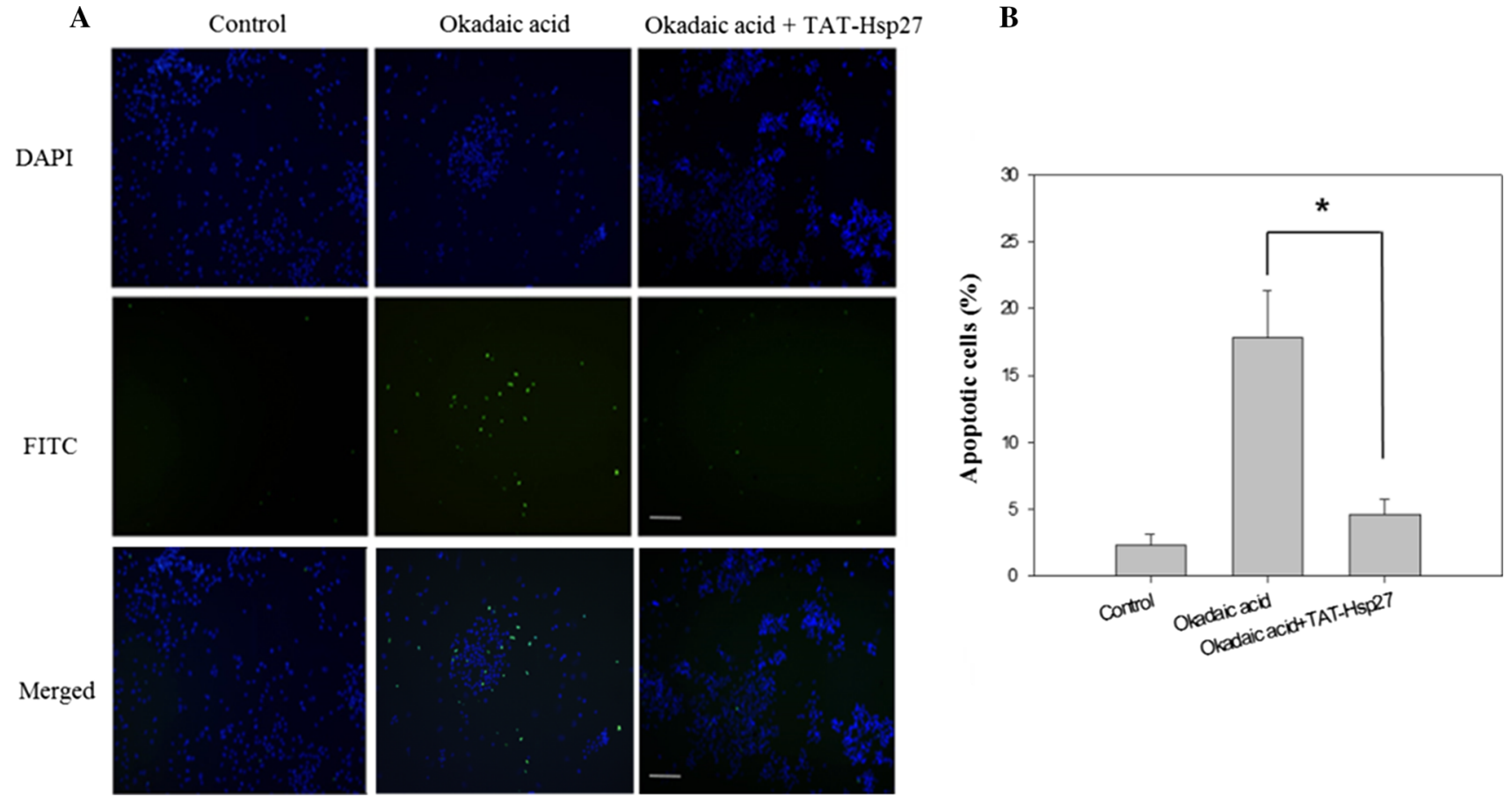

Fig. 6 Tat-Hsp27 inhibits apoptotic cell death induced by okadaic acid. The TUNEL assay was performed to determine the extent of programmed cell death. SH-SY5Y cells were treated with $10 \mathrm{nM}$ okadaic acid alone or following pretreatment with $2 \mu \mathrm{M}$ Tat-Hsp27. a Representative images of the TUNEL assay. Scale bar $100 \mu \mathrm{m}$. b Quantitation of the percentage of TUNEL-positive cells indicated that apoptosis induced by okadaic acid was decreased in cells pretreated with Tat-Hsp27. Values indicate mean \pm SD (Anova, Tukey's HSD test, $n=3$ per group). ${ }^{*} p<0.05$

Therefore, we anticipate that the uptake of Tat-Hsp27 into the brain will readily occur when nasally administered and will protect against cell death caused by hyperphosphorylated tau, which is one of the primary causes of $\mathrm{AD}$ tein to treat a cellular AD model, and this fusion protei was delivered into cells at high levels within 2 h (Fig. 2). 
(Brasnjevic et al. 2009; Schwarze et al. 1999). Our results suggest that Tat-Hsp27 has a protective effect against hyperphosphorylated tau and could represent a valuable protein therapeutic for AD.

Acknowledgments This study was supported by a grant from the Basic Science Research Program through a National Research Foundation of Korea (NRF) funded by the Korean government (2012K001411)

Conflict of interest All authors state that they have no conflicts of interest.

Open Access This article is distributed under the terms of the Creative Commons Attribution 4.0 International License (http:// creativecommons.org/licenses/by/4.0/), which permits unrestricted use, distribution, and reproduction in any medium, provided you give appropriate credit to the original author(s) and the source, provide a link to the Creative Commons license, and indicate if changes were made.

\section{References}

Ahn J, Kim H, Park J-S (2013) Hsp27 reduces phosphorylated tau and prevents cell death in the human neuroblastoma cell line $\mathrm{SH}$ SY5Y. Bull Korean Chem Soc 34:1503-1507

Andorfer C, Kress Y, Espinoza M, Silva RD, Tucker KL, Barde Y-A, Duff K, Davies P (2003) Hyperphosphorylation and aggregation of tau in mice expressing normal human tau isoforms. J Neurochem 86:582-590

Arrigo A-P (2013) Human small heat shock proteins: protein interactomes of homo- and hetero-oligomeric complexes: an update. FEBS Lett 587:1959-1969

Baglietto-Vargas D, Kitazawa M, Le EJ, Estrada-Hernandez T, Rodriguez-Ortiz CJ, Medeiros R, Green K, LaFerla FM (2014) Endogenousmurine tau promotes neurofibrillary tangles in $3 \times \mathrm{Tg}$ AD mice without affecting cognition. Neurobiol Dis 62:407-415

Becker-Hapak M, McAllister SS, Dowdy SF (2001) TAT-mediated protein transduction into mammalian cells. Methods 24:247-256

Brasnjevic I, Steinbusch HWM, Schmitz C, Martinez-Martinez P (2009) Delivery of peptide and protein drugs over the bloodbrain barrier. Prog Neurobiol 87:212-251

Buée L, Bussiére T, Buée-Scherrer VR, Delacourte A, Hof PR (2000) Tau protein isoforms, phosphorylation and role in neurodegenerative disorders. Brain Res Rev 33:95-130

Carrettiro Daniel C, Hernandez Israel, Neveu Pierre, Papagiannakopoulos Thales, Kosik Kenneth S (2009) The cochaperone BAG2 sweeps paired helical filament-insoluble tau form the microtuble. J Neurosci 29(7):2151-2161

Chabrier MA, Cheng D, Castello NA, Green KN, LaFerla FM (2014) Synergistic effects of amyloid-beta and wild-type human tau on dendritic spine loss in a floxed double transgenic model of Alzheimer's disease. Neurobiol Dis 64:107-117

Citron M (2010) Alzheimer's disease: strategies for disease modification. Nat Rev Drug Discov 9:387-398

Concannon CG, Gorman AM, Samali A (2003) On the role of Hsp27 in regulating apoptosis. Apoptosis 8:61-70

Crimins JL, Pooler A, Polydoro M, Luebke JI, Spires-Jones TL (2013) The intersection of amyloid beta and tau in glutamatergic synaptic dysfunction and collapse in Alzheimer's disease. Ageing Res Rev 12:757-763
Denk F, Wade-Martins R (2009) Knock-out and transgenic mouse models of tauopathies. Neurobiol Aging 30:1-13

Goedert M, Jakes R, Vanmechelen E (1995) Monoclonal antibody AT8 recognises tau protein phosphorylated at both serine 202 and threonine 205. Neurosci Lett 189:167-170

Götz J, Chen F, Dorpe JV, Nitsch RM (2001) Formation of neurofibrillary tangles in $\mathrm{P} 301 \mathrm{~L}$ tau transgenic mice induced by A $\beta 42$ fibrils. Science 293:1491-1495

Green I, Christison R, Voyce CJ, Bundell KR, Lindsay MA (2003) Protein transduction domains: are they delivering? Trends Pharmacol Sci 24:213-215

Hernandez F, Avila J (2007) Tauopathies. Cell Mol Life Sci 64:2219-2233

Jakob U, Gaestel M, Engel K, Buchner J (1993) Small heat shock proteins are molecular chaperones. J Biol Chem 268:1517-1520

Kamat PK, Rai S, Nath C (2013) Okadaic acid induced neurotoxicity: an emerging tool to study Alzheimer's disease pathology. Neurotoxicology 37:163-172

Kosik KS, Shimura H (2005) Phosphorylated tau and the neurodegenerative foldopathies. Biochim Biophys Acta 1739:298-310

Kwon JH, Kim J-B, Lee K-H, Kang S-M, Chung N, Jang Y, Chung JH (2007) Protective effect of heat shock protein 27 using protein transduction domain-mediated delivery on ischemia/ reperfusion heart injury. Biochem Biophys Rea Commun 363:399-404

Medeiros R, Baglietto-Vargas D, LaFerla FM (2010) The role of tau in Alzheimer's disease and related disorders. CNS Neurosci Ther 17:514-524

Ozsoy Y, Gungor S, Cevher E (2009) Nasal delivery of high molecular weight drugs. Molecules 14:3754-3779

Patil Sachin P, Tran Nhung, Geekiyanage Hirosha, Liu Li, Chan Christina (2013) Cucumin-induced upregulation of the abti-tau cochaperone BAG2 in primary rat cortical neurons. Neurosci Lett 554:121-125

Read DE, Gorman AM (2009) Heat shock protein 27 in neuronal survival and neurite outgrowth. Biochem Biophys Res Commun 382:6-8

Rogers M-L, Rush RA (2012) Non-viral gene therapy for neurological diseases, with an emphasis on targeted gene delivery. J Controll Release 157:183-189

Saidi Laiq-Jan, Polydoro Manuela, Kay Kevin R, Sanchez Laura, Mandelkow Eva-Maria, Hyman Bradley T, Spires-Jones Tara L (2015) Carboxy terminus heat shock protein 70 interacting protein reduces tau-associated degenerative changes. J Alzheimers Dis 44:937-947

Salminen A, Ojala J, Kaarniranta K, Hiltunen M, Soininen H (2011) Hsp90 regulates tau pathology through co-chaperone complexes in Alzheimer's disease. Prog Neurobiol 93:99-110

Santa-Maria I, Varghese M, Ksieżak-Reding H, Dzhun A, Wang J, Pasinetti GM (2012) Paired helical filaments from Alzheimer disease brain induce intracellular accumulation of tau protein in aggresomes. J Biol Chem 287:20522-20533

Schwarze SR, Ho A, Vocero-Akbani A, Dowdy SF (1999) In vivo protein transduction: delivery of a biologically active protein into the mouse. Science 285:1569-1572

Selkoe DJ (2002) Alzheimer's disease is a synaptic failure. Science 298:789-791

Shimura H, Miura-Shimura Y, Kosik KS (2004a) Binding of tau to heat shock protein 27 leads to decreased concentration of hyperphosphorylated tau and enhanced cell survival. J Biol Chem 279:17957-17962

Shimura H, Schwartz D, Gygi SP, Kosik KS (2004b) CHIP-Hsc70 complex ubiquitinates phosphorylated tau and enhances cell survival. J Biol Chem 279:4869-4876

Song IS, Kang S-S, Kim E-S, Park H-M, Choi CY, Tchah H, Kim JY (2014) Heat shock protein 27 phosphorylation is involved in 
epithelial cell apoptosis as well as epithelial migration during corneal epithelial wound healing. Exp Eye Res 118:36-41

Stetler RA, Gan Y, Zhang W, Liou AK, Gao Y, Cao G, Chen J (2010) Heat shock proteins: cellular and molecular mechanisms in the central nervous system. Prog Neurobiol 92:184-211

Wadia JS, Dowdy SF (2005) Transmembrane delivery of protein and peptide drugs by TAT-mediated transduction in the treatment of cancer. Adv Drug Deliv Rev 57:579-596
Wischik CM, Harrington CR, Storey JMD (2014) Tau-aggregation inhibitor therapy for Alzheimer's disease. Biochem Pharmacol 88:529-539

Zhang Z, Simpkins JW (2010) Okadaic acid induces tau phosphorylation in SH-SY5Y cells in an estrogen-preventable manner. Brain Res 1345:176-181 